\title{
CORRIGENDUM
}

\section{CHEK2 genomic and proteomic analyses reveal genetic inactivation or endogenous activation across the 60 cell lines of the US National Cancer Institute}

\author{
G Zoppoli, S Solier, WC Reinhold, H Liu, JW Connelly Jr, A Monks, RH Shoemaker,
} OD Abaan, SR Davis, PS Meltzer, JH Doroshow and Y Pommier

Oncogene (2012) 31, 535-536; doi:10.1038/onc.2011.420

Correction to: Oncogene (2012) 31, 403-418; doi:10.1038/ onc.2011.283; published online 18 July 2011

Figure $5 \mathrm{~b}$ (left panel) has been corrected. The arrows have been repositioned for some of the cell lines to indicate their p53 wild-type status based on our published data (CellMiner, http://discover.nci.nih.gov/ cellminer/mutationGeneLoad.do, and ongoing exome sequencing of the NCI60).
This change strengthens our conclusions that endogenously activated Chk2 (pT68-Chk2) is only observed in p53-defective cells. All the 16 cell lines with wild-type p53 were negative for activated Chk2 whereas the 8 cell lines (including HeLa) with activated Chk2 were all p53 defective $(P<0.05$, $\chi^{2}$, two-sided).

The Corrected Figure 5 is shown on the next page. 
a

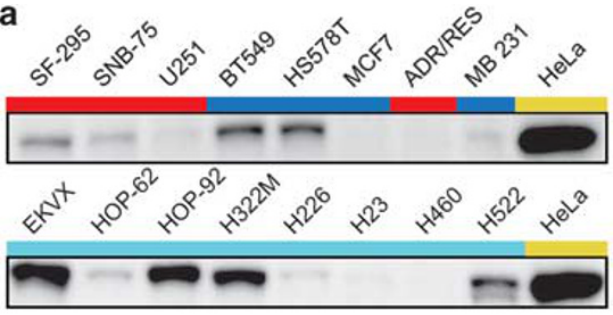

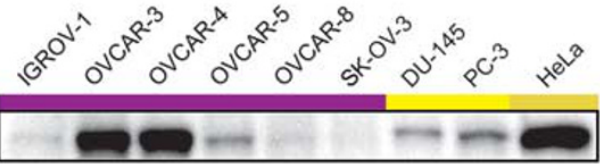

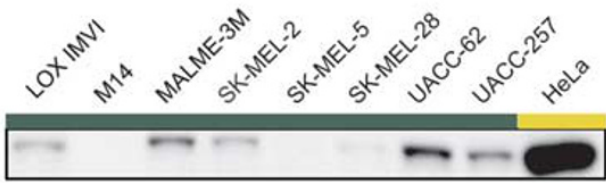

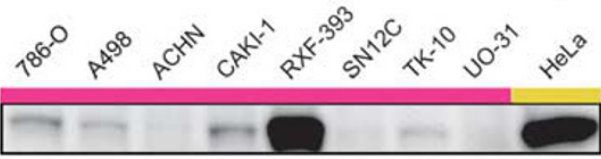
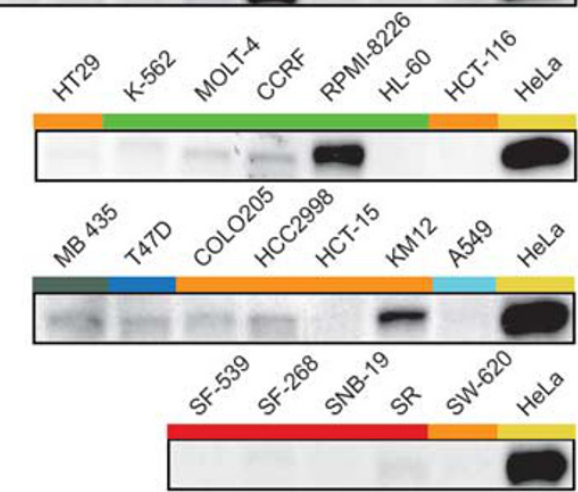

Leukemia

Melanoma

Lung b

p-T68 Chk2

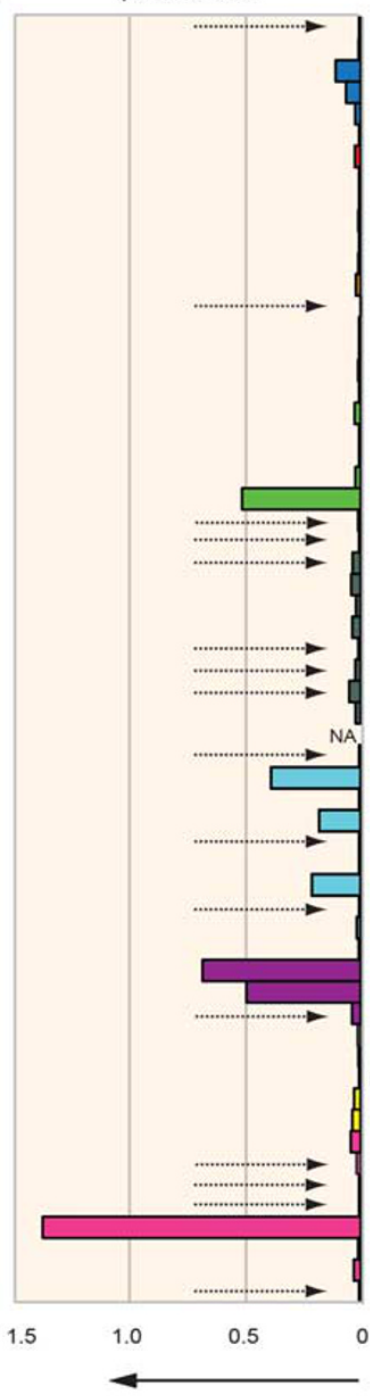

MCF7

MCF7 231

HS578T

BT549

T47D

SF-268

SF-295

SF. 539

SNB-19

SNB-75

U 251

COLO205

HCC 2998

HCT-116

HCT-15

HT29

KM12
SW-620

SW-620

HL60

K-562

MOLT-4
RPMI-8226

SR

LOXIMVI

MALME $3 \mathrm{M}$

SK MEL-2

SK MEL-5

(2)

UACC-62

MDAN

A549

EKVX

HOP 62
HOP 92

HOP 92

$\mathrm{NCl} \mathrm{H226}$



$\mathrm{NCl} \mathrm{H} 460$

$\mathrm{NCl} \mathrm{H522}$

IGROV1

OVCAR 3

OVCAR 4

OVCAR 5

OVCAR 8

I ADR RES

PC 3

DU 145

7860

A498

ACHN
CAKI 1

RXF 393

SN12C

TK 10

UO 31

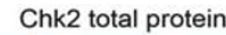

Chk2 total prot

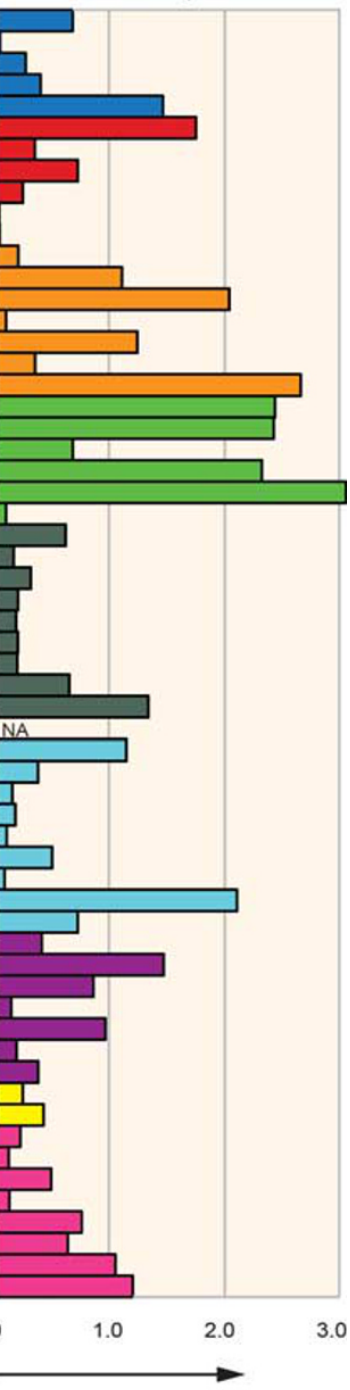

Figure 5 Proteomic analysis of total and phosphorylated (pT68) Chk2 shows high endogenous levels of Chk2 activation in several cancer cell lines across the NCI-60. (a) Representative western blot pictures of pT68-Chk2 in the NCI-60. The colored bars correspond to the tissues of origin (see legend at the bottom). HeLa cells were used as positive control and to calculate the Chk2 phosphorylation ratios in the NCI-60. (b) Quantitation of pT68-Chk2 (left) and total Chk2 (right) protein levels in the NCI-60. The colored bars represent the means of the ratios between the western blot intensities of the individual cell lines and the HeLa reference loading, after normalization by $\beta$-actin loading. The colors represent the tissues of origin (see also Figure 1). The dotted arrows indicate known p53 wild-type cancer cell lines. NA: not available. 